\title{
Surveillance accrue des méningococcies invasives au Canada, 2006-2011
}

\author{
Li YA ${ }^{1^{\star}}$, Tsang $\mathbf{R}^{2}$, Desai $\mathbf{S}^{1}$ et Deehan $\mathrm{H}^{1}$ \\ Agence de la santé publique du Canada, Centre de l'immunisation et des maladies respiratoires infectieuses, Ottawa \\ (Ontario) \\ 2 Agence de la santé publique du Canada, Laboratoire national de microbiologie, Winnipeg (Manitoba) \\ * Auteur-ressource : y.anita.li@phac-aspc.gc.ca
}

\section{Résumé}

Objectif : L'objectif du présent rapport est de décrire l'épidémiologie de la méningococcie invasive au Canada de 2006 à 2011.

Méthodes : Les données obtenues à partir du Système national de surveillance améliorée de la méningococcie invasive et les estimations démographiques nationales ont été sélectionnées aux fins d'analyses descriptives et inférentielles. Les répartitions géographiques, démographiques, saisonnières et par sous-types ainsi que les caractéristiques cliniques des cas de méningococcie invasive ont été examinées. Les taux d'incidence et de mortalité ont été calculés pour 100000 habitants par année; des intervalles de confiance (IC) à $95 \%$ ont été calculés aux fins de comparaison des taux. La méthode directe a été utilisée pour la standardisation de l'âge. Les proportions ont été comparées à l'aide du test de chi carré, avec un niveau d'importance de $p<0,05$.

Résultats : Au cours de la période de l'étude, les taux d'incidence moyens de méningococcie invasive étaient de 0,58 (total), 0,33 (sérogroupe B), 0,07 (sérogroupe $C$ ), 0,03 (sérogroupe $W$-135) et de 0,10 (sérogroupe $Y$ ). L'âge médian pour les sérogroupes $B, C, W-135$ et $Y$ était de 16, 43, 38 et 47 ans, respectivement. Les taux d'incidence moyens propres à l'âge chez les nourrissons de moins de 1 an $(7,35, I C: 5,38-9,32)$ et les enfants de 1 à 4 ans $(1,89, I C: 1,54-2,24)$ étaient nettement supérieurs à ceux d'autres groupes d'âge. Le rapport moyen de mortalité était de $8,1 \%$ (plage de $4,3 \%$ à $14,3 \%$ ). Le nombre moyen mensuel de cas était nettement plus élevé ( $p<0,0001)$ en hiver (18 cas) qu'en été (12 cas).

Conclusion : La méningococcie invasive est toujours endémique au Canada. Bien que les personnes de tout âge puissent être touchées, les nourrissons de moins de 1 an sont les plus à risque, suivis des enfants de 1 à 4 ans et des personnes de 15 à 19 ans. Après la mise en œuvre de programmes d'immunisation systématique des enfants à l'aide de vaccins conjugués monovalents contre le méningocoque du groupe $C$ dans l'ensemble des provinces et territoires (depuis 2007), l'incidence du méningocoque du groupe $C$ a nettement diminué au cours de la période de l'étude, et se trouve actuellement à son plus bas niveau historique. Le sérogroupe $B$ est la principale cause de méningococcie invasive, et les taux d'incidence de maladies associées aux sérogroupes $\mathrm{W}-135$ et $\mathrm{Y}$ se sont stabilisés à des niveaux relativement plus bas. Compte tenu de l'ajout de programmes d'immunisation utilisant des vaccins quadrivalents conjugués contre le méningocoque, l'on pourrait s'attendre à une réduction supérieure de l'incidence d'infections à méningocoque au Canada.

\section{Introduction}

La méningococcie invasive est causée par Neisseria meningitidis, une bactérie Gram-négative. Les bactéries Neisseria sont classées en deux sous-types, en fonction de la réactivité immunologique de leur capsule polysaccharidique (1). L'évolution de la méningococcie invasive est souvent rapide et se manifeste généralement sous forme de septicémie ou de méningite (2). La méningococcie invasive est une maladie à déclaration obligatoire au Canada depuis 1924 (3), conformément au Système canadien de surveillance des maladies à déclaration obligatoire, fournissant uniquement des données épidémiologiques de base. Au Canada, les cas de méningococcie invasive attribuables aux sérogroupes $A$ et $C$ étaient le plus souvent observés avant 1975, et ceux attribuables au sérogroupe B, entre 1975 et 1985 (4). Depuis 1985, le nombre total de cas de méningococcie 
invasive et le nombre de cas associés au sérogroupe $C$ ont augmenté, atteignant un sommet en $1992(5,6)$. Par conséquent, le Système national de surveillance améliorée de la méningococcie invasive a été établi en 1992 au Canada, afin de consigner des données épidémiologiques et de laboratoire. Des programmes d'immunisation systématique à l'aide du vaccin conjugué contre le méningocoque $C$, recommandés pour les enfants par le Comité consultatif national de l'immunisation (6), ont été mis en œuvre au Canada depuis 2002 (7). De 2002 à 2005 , époque où seulement quelques provinces et territoires canadiens avaient mis en œuvre des programmes d'immunisation à l'aide du vaccin conjugué contre le méningocoque $\mathrm{C}$, l'incidence de la maladie due au sérogroupe $\mathrm{C}$ a diminué de $43 \%$, comparativement à la période de 1995 à 2001 (8). L'information au sujet de la méningococcie invasive au Canada est publiée par l'Agence de la santé publique du Canada (I'Agence) depuis 1979 (4,8-18). Le dernier rapport de surveillance publié par l'Agence a fourni une description de la méningococcie invasive au Canada, de 2004 à 2005 (8). Le rapport suivant décrit l'épidémiologie de la méningococcie invasive au Canada, du $1^{\text {er }}$ janvier 2006 au 31 décembre 2011.

\section{Méthodes}

\section{Données de surveillance}

Le présent rapport est fondé sur des données au sujet de la méningococcie invasive, tirées du Système national de surveillance améliorée de la méningococcie invasive, portant sur l'apparition de la maladie entre le $1^{\text {er }}$ janvier 2006 et le 31 décembre 2011. Selon la définition nationale de cas (3), la surveillance au cours de cette période a relevé des données au sujet de cas confirmés et de cas probables. Les ministères de la Santé des provinces et territoires signalent des données épidémiologiques non nominatives à l'Agence, portant sur tous les cas de méningococcie invasive correspondant à la définition de cas nationale. Les laboratoires de santé publique ou d'hôpitaux des provinces et territoires envoient des isolats de cas de $N$. meningitidis au Laboratoire national de microbiologie afin qu'il confirme le sérogroupe et effectue d'autres analyses bactériologiques. Ces analyses consistent en des sérotypes et sous-types pour tous les isolats, électrophorèses enzymatiques multilocus ou typages génomiques multilocus, pour tous les isolats appartenant au sérogroupe $C$ (16), et, plus récemment, les typages génomiques multilocus pour des isolats liés à des éclosions ou aux fins d'analyses spéciales. Un appariement probabiliste a été effectué afin de coupler des données épidémiologiques et des données de laboratoire, à l'aide des variables suivantes : province/territoire, date de naissance ou âge, sexe, date d'apparition de la maladie et sérogroupe.

Les estimations de population pour les provinces et territoires ont été obtenues de Statistique Canada et sont fondées sur les données du recensement de 2006 (19). La population canadienne de 1991 a été choisie comme population stable pour la standardisation de l'âge. La répartition de la population est fondée sur les estimations finales de la population canadienne postcensitaire du $1^{\mathrm{er}}$ juillet 1991, ajustée en fonction du sous-dénombrement du recensement. La répartition selon l'âge de la population a été pondérée et normalisée (20).

Les données utilisées dans cette étude sont tirées de la surveillance de la santé publique, et sont dispensées de l'approbation du comité d'examen éthique.

\section{Analyse statistique}

Les répartitions géographiques, démographiques, saisonnières et par sous-types, ainsi que les caractéristiques cliniques des cas de méningococcie invasive ont été examinées. Les taux d'incidence ont été calculés à l'aide des données de 2006-2011, tirées du Système national de surveillance améliorée de la méningococcie invasive et d'estimations démographiques. Tous les taux d'incidence et de mortalité ont été calculés pour 100000 habitants par année. La méthode directe a été utilisée pour le calcul des taux standardisés en fonction de l'âge. Les cas pour lesquels l'âge n'était pas disponible ont été exclus des calculs de standardisation de l'âge et de toutes les analyses liées à l'âge. Les intervalles de confiance pour les taux d'incidence ont été calculés selon un niveau de confiance de $95 \%$. Les intervalles de confiance des taux standardisés en fonction de l'âge ont été calculés selon la méthode fondée sur la distribution gamma (21). Les intervalles de confiance des taux d'incidence bruts et propres à l'âge ont été calculés à l'aide de méthodes fondées sur la distribution de Poisson pour les nombres de cas inférieurs à 100, et sur la distribution normale pour les nombres de cas égaux ou supérieurs à 100. Les 
proportions ont été comparées à l'aide du test de chi carré, avec un niveau d'importance de $p<0,05$. Pour les analyses saisonnières, la période hivernale a été définie de novembre à avril, et la période estivale, de mai à octobre. Des analyses descriptives et inférentielles ont été menées à l'aide de Microsoft Excel 2010 et de SAS EG v. 5.1.

\section{Résultats}

\section{Aperçu}

De 2006 à 2011, un total de 1174 cas de méningococcie invasive ont été signalés au Canada, à raison de 154 à 229 cas par année (Figure 1). À l'exception de 21 cas pour lesquels des données étaient manquantes, 1121 cas (97\%) ont été confirmés en laboratoire et 32 (3\%) étaient des cas probables. Parmi les cas confirmés en laboratoire, $777(68 \%)$ se sont avérés positifs aux termes d'une culture bactérienne, $111(10 \%)$, par réaction en chaîne de la polymérase (RCP) et $44(4 \%)$, par culture bactérienne et RCP; dans les autres cas, la méthode de laboratoire n'a pas été précisée.

Figure 1 : Nombre de cas de méningococcie invasive et taux d'incidence globaux (par 100000 habitants) au Canada, par année, 2006-2011*

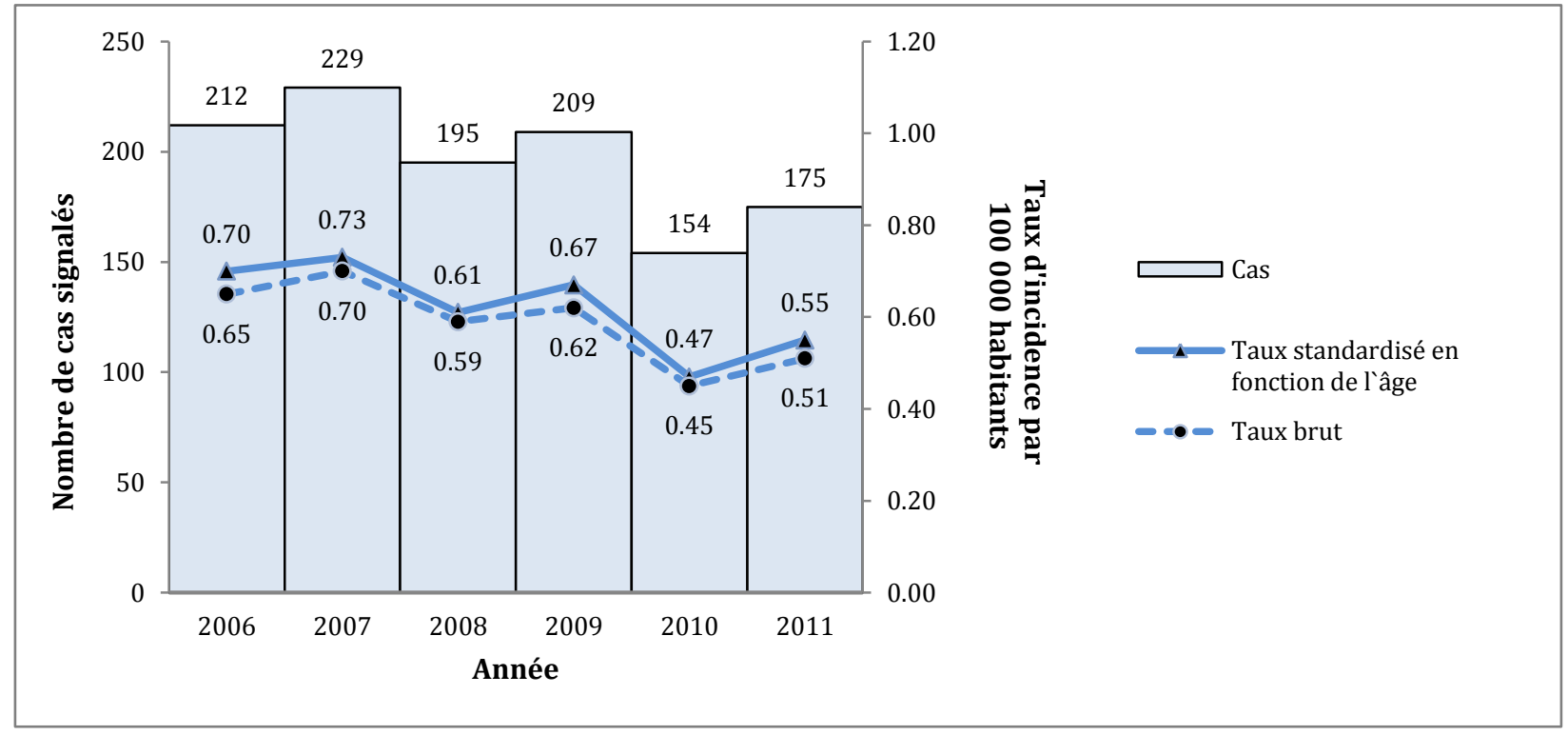

*Trois cas pour lesquels des données sur l'âge étaient manquantes ont été exclus dans le calcul des taux standardisés en fonction de l'âge.

Trois cas pour lesquels des données sur l'âge étaient manquantes ont été exclus des calculs de taux standardisés en fonction de l'âge et autres analyses liées à l'âge. Le Tableau 1 fournit des taux d'incidence bruts détaillés et des taux standardisés en fonction de l'âge. Les taux d'incidence moyens bruts et standardisés en fonction de l'âge étaient de 0,58 et de 0,60 , respectivement. Le taux d'incidence standardisé en fonction de l'âge de méningococcie invasive en $2010(0,47, I C: 0,39-0,54)$ était nettement inférieur qu'en $2006(0,70$, IC : 0,61-0,80), $2007(0,73$, IC : 0,63-0,82) et $2009(0,67$, IC : 0,58-0,76). Aucune différence importante n'a été relevée dans les comparaisons d'autres années. En raison du faible nombre de cas de méningococcie invasive des sérogroupes $A, X, Z$ et $29 \mathrm{E}$, ils ont été regroupés sous le sérogroupe " Autres » aux fins d'analyses subséquentes. 
Tableau 1 :Taux d'incidence bruts et standardisés en fonction de l'âge (par 100000 habitants) de méningococcie invasive au Canada, par sérogroupe et année, 2006-2011*

\begin{tabular}{|c|c|c|c|c|c|c|c|c|}
\hline \multirow{2}{*}{$\begin{array}{l}\text { Type de } \\
\text { taux }\end{array}$} & \multirow{2}{*}{ Sérogroupe } & \multicolumn{6}{|c|}{ Année } & \multirow{2}{*}{$\begin{array}{c}\text { Moyenne (IC à } 95 \%) \\
2006-2011\end{array}$} \\
\hline & & 2006 & 2007 & 2008 & 2009 & 2010 & 2011 & \\
\hline \multirow[t]{8}{*}{ Brut } & Total & 0,65 & 0,70 & 0,59 & 0,62 & 0,45 & 0,51 & $0,58(0,49-0,68)$ \\
\hline & B & 0,35 & 0,40 & 0,29 & 0,37 & 0,27 & 0,31 & $0,33(0,28-0,38)$ \\
\hline & C & 0,13 & 0,09 & 0,09 & 0,06 & 0,03 & 0,01 & $0,07(0,02-0,12)$ \\
\hline & W-135 & 0,02 & 0,04 & 0,04 & 0,04 & 0,02 & 0,03 & $0,03(0,02-0,04)$ \\
\hline & $\mathbf{Y}$ & 0,08 & 0,11 & 0,11 & 0,09 & 0,08 & 0,10 & $0,10(0,09-0,11)$ \\
\hline & Autres $^{\dagger}$ & 0,006 & 0,006 & 0,003 & 0,018 & 0,006 & 0,012 & $0,008(0,003-0,014)$ \\
\hline & Non sérogroupable & 0,006 & 0,006 & 0,006 & 0,003 & 0,006 & 0,003 & $0,005(0,003-0,007)$ \\
\hline & Inconnu & 0,06 & 0,05 & 0,04 & 0,04 & 0,03 & 0,03 & $0,04(0,03-0,05)$ \\
\hline \multirow{8}{*}{$\begin{array}{l}\text { Standardisé } \\
\text { en fonction } \\
\text { de l'âge }\end{array}$} & Total & 0,70 & 0,73 & 0,61 & 0,67 & 0,47 & 0,55 & $0,60(0,47-0,73)$ \\
\hline & B & 0,40 & 0,44 & 0,33 & 0,40 & 0,24 & 0,36 & $0,36(0,29-0,43)$ \\
\hline & C & 0,14 & 0,09 & 0,08 & 0,06 & 0,03 & 0,01 & $0,07(0,02-0,11)$ \\
\hline & W-135 & 0,02 & 0,04 & 0,04 & 0,04 & 0,02 & 0,03 & $0,03(0,02-0,04)$ \\
\hline & $\mathbf{Y}$ & 0,08 & 0,10 & 0,10 & 0,09 & 0,07 & 0,09 & $0,09(0,08-0,10)$ \\
\hline & Autres $^{\dagger}$ & 0,005 & 0,005 & 0,004 & 0,013 & 0,005 & 0,011 & $0,007(0,003-0,011)$ \\
\hline & Non sérogroupable & 0,007 & 0,006 & 0,007 & 0,002 & 0,005 & 0,002 & $0,005(0,003-0,007)$ \\
\hline & Inconnu & 0,06 & 0,05 & 0,04 & 0,03 & 0,02 & 0,02 & $0,04(0,02-0,05)$ \\
\hline
\end{tabular}

*Au total, 82 cas dont les sérogroupes étaient manquants ont été exclus des calculs de taux d'incidence bruts. Au total, 83 cas dont le sérogroupe ou l'âge étaient manquants ont été exclus des calculs de taux d'incidence standardisés en fonction de l'âge.

†Autres : sérogroupes $A, X, Z$ et $29 E$.

\section{Répartition géographique}

Comme le montre la Figure 2, des cas de méningococcie invasive ont été signalés dans tous les territoires et provinces du Canada, mais pas toutes les années de la période de l'étude. Le Nunavut présentait le taux d'incidence standardisé en fonction de l'âge le plus élevé, à savoir, de 4,09, en 2006. Puisque l'île-du-PrinceÉdouard et les trois territoires (Yukon, Nunavut et les Territoires du Nord-Ouest) avaient signalé des cas seulement au cours d'une ou deux années et que les populations de ces territoires sont faibles, il n'est pas approprié de comparer leurs taux d'incidence moyens standardisés en fonction de l'âge aux taux d'autres provinces. Le taux d'incidence moyen standardisé en fonction de l'âge du Québec $(1,12, I C: 0,95-1,29)$ était nettement plus élevé que celui de toutes les autres provinces, à l'exception de Terre-Neuve-et-Labrador $(1,08$, IC : 0,53-1,64) et du Nouveau-Brunswick $(0,87$, IC : 0,57-1,17). À titre de comparaison, les autres provinces présentaient des taux d'incidence moyens standardisés en fonction de l'âge variés, mais pas de façon significative. 
Figure 2 : Taux d'incidence standardisés en fonction de l'âge (par 100000 habitants) de méningococcie invasive au Canada, par province/territoire, 2006-2011**

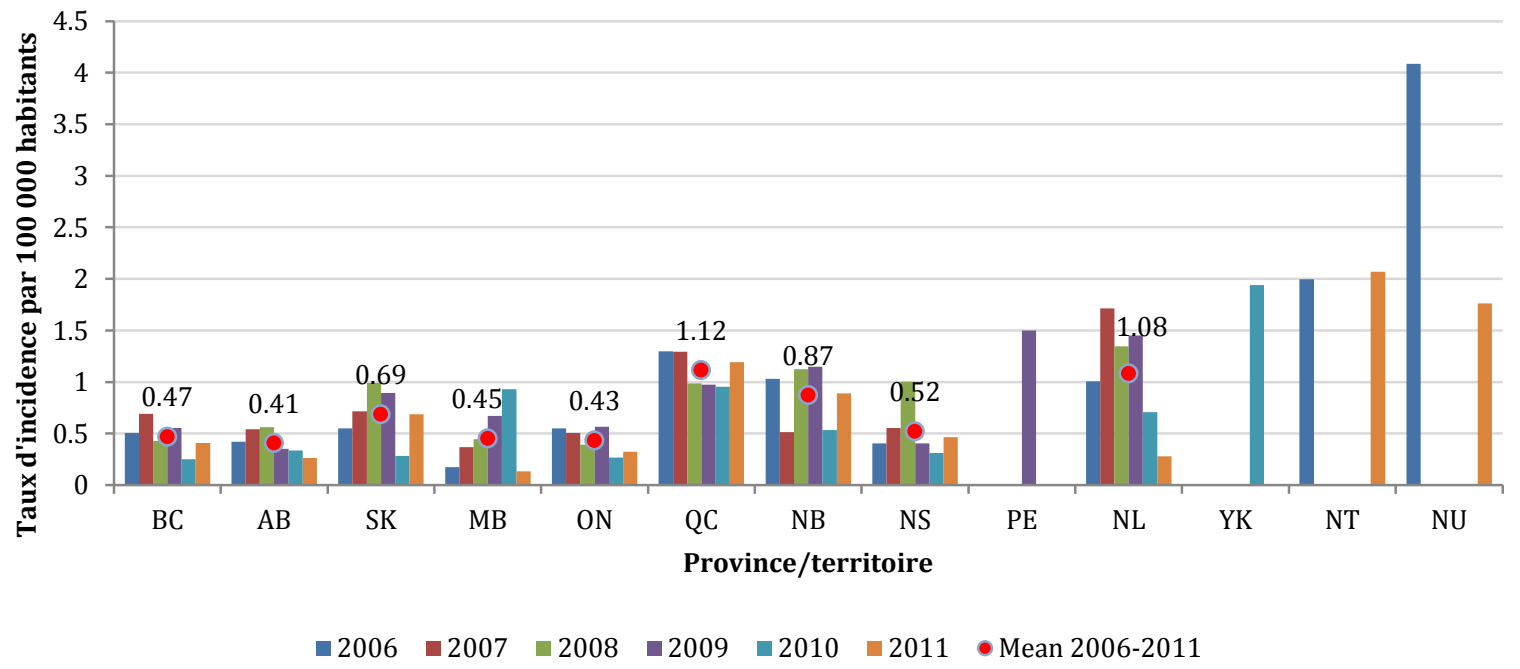

*Trois cas pour lesquels des données sur l'âge étaient manquantes ont été exclus. Puisque l'île-du-Prince-Édouard et les trois territoires (Yukon, Nunavut et les Territoires du Nord-Ouest) avaient signalé des cas seulement au cours d'une ou deux années et que les populations de ces territoires sont faibles, il n'est pas approprié de comparer leurs taux d'incidence moyens standardisés en fonction de l'âge aux taux d'autres provinces. Par conséquent, les taux d'incidence moyens standardisés en fonction de l'âge pour ces quatre provinces et territoires n'ont pas été affichés.

\section{Répartition démographique}

Pour tous les cas signalés, l'âge variait de moins d'un mois à 98 ans, et l'âge médian était de 20 ans. L'étendue de l'âge médian était de 18 à 23 ans, pour la période de l'étude. La répartition selon l'âge était variée parmi les différents sérogroupes de cas de méningococcie (Figure 3). L'âge médian pour les sérogroupes B, C, W-135 et $Y$ était de 16, 43, 38 et 47 ans, respectivement. Bien qu'ils ne constituent que $6 \%$ de la population totale, les enfants âgés de moins de 5 ans représentaient $37 \%$ des cas d'infections par le sérogroupe $B, 31 \%$ par le sérogroupe $\mathrm{W}-135$, mais seulement $9 \%$ par le sérogroupe $C$ et $10 \%$ des cas pour le sérogroupe $\mathrm{Y}$. Les personnes âgées de plus de 40 ans ont constitué la majorité des cas d'infection par le sérogroupe C (51\%) et le sérogroupe $Y(59 \%)$, près de la moitié des cas d'infection par le sérogroupe $\mathrm{W}-135$ (48 \%), mais seulement $21 \%$ des cas d'infection par le sérogroupe B. 
Figure 3 : Répartition selon l'âge des cas de méningococcie invasive, par sérogroupe, 2006-2011*

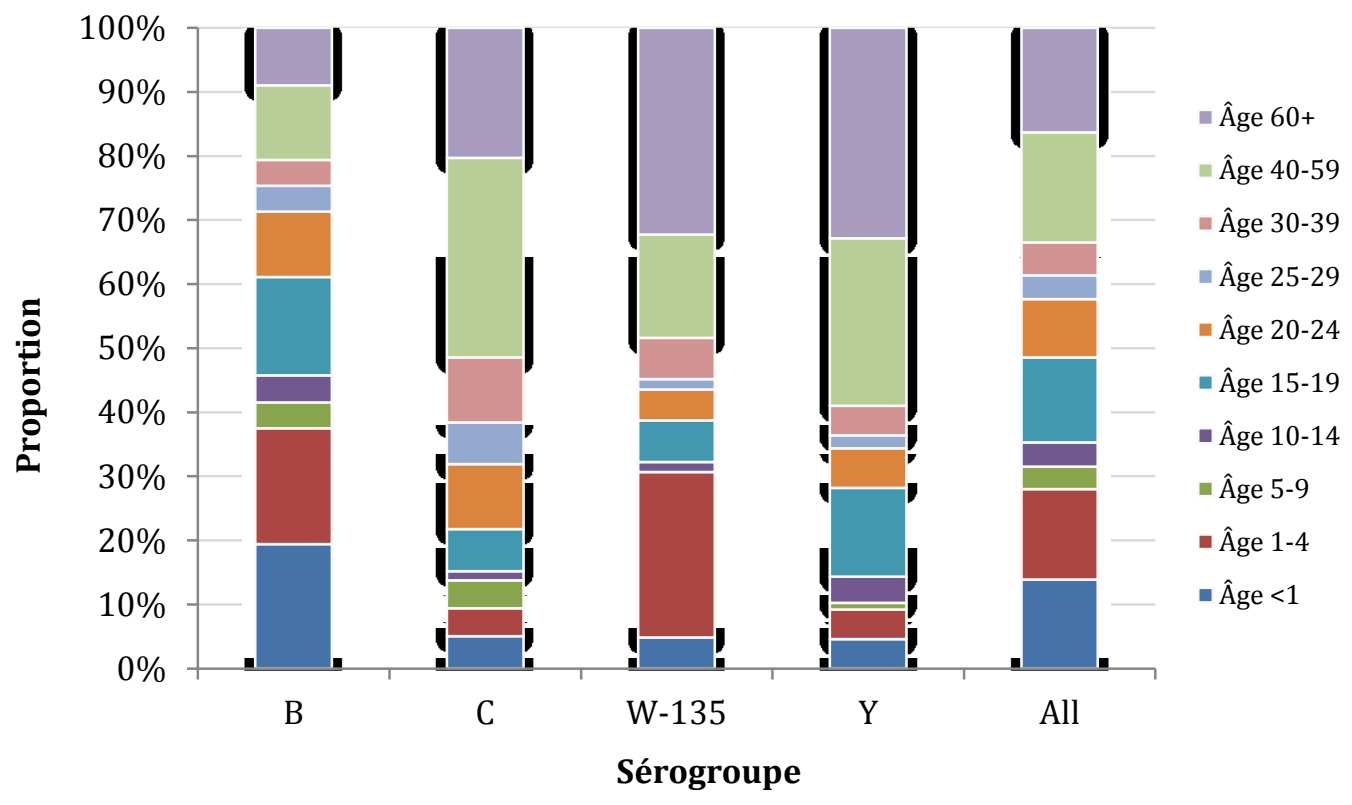

*Au total, 83 cas dont le sérogroupe ou l'âge étaient manquants ont été exclus.

Comme le montre le Tableau 2, les taux d'incidence moyens propres à l'âge chez les nourrissons de moins de 1 an $(7,35$, IC : 5,38-9,32) et les enfants de 1 à 4 ans $(1,89$, IC : 1,54-2,24) étaient nettement supérieurs à ceux de tous les autres groupes d'âge. Les adolescents de 15 à 19 ans présentaient le troisième taux d'incidence le plus élevé $(1,17$, IC : 1,00-1,34), sauf pour la tranche d'âge de 20 à 24 ans $(0,77, I C: 0,48-1,05)$.

Tableau 2 : Taux d'incidence propre à l'âge (par 100000 habitants) de méningococcie invasive au Canada, par année, 2006-2011*

\begin{tabular}{|c|c|c|c|c|c|c|c|}
\hline \multirow{2}{*}{$\begin{array}{l}\text { Groupe } \\
\text { d'âge }\end{array}$} & \multicolumn{6}{|c|}{ Année } & \multirow{2}{*}{$\begin{array}{l}\text { Moyenne (IC : } 95 \% \text { ) } \\
2006-2011\end{array}$} \\
\hline & 2006 & 2007 & 2008 & 2009 & 2010 & 2011 & \\
\hline Tous & 0,65 & 0,70 & 0,59 & 0,62 & 0,45 & 0,51 & $0,58(0,49-0,68)$ \\
\hline$<1$ & 7,71 & 6,94 & 6,97 & 10,57 & 4,75 & 7,15 & $7,35(5,38-9,32)$ \\
\hline $1-4$ & 1,81 & 1,92 & 1,61 & 2,32 & 1,46 & 2,22 & $1,89(1,54-2,24)$ \\
\hline $5-9$ & 0,44 & 0,78 & 0,22 & 0,50 & 0,11 & 0,22 & $0,38(0,12-0,63)$ \\
\hline $10-14$ & 0,43 & 0,34 & 0,45 & 0,35 & 0,26 & 0,37 & $0,37(0,30-0,44)$ \\
\hline $15-19$ & 1,40 & 1,30 & 1,20 & 1,02 & 0,99 & 1,09 & $1,17(1,00-1,34)$ \\
\hline $20-24$ & 1,24 & 0,92 & 0,70 & 0,60 & 0,63 & 0,50 & $0,77(0,48-1,05)$ \\
\hline $25-29$ & 0,27 & 0,54 & 0,35 & 0,30 & 0,21 & 0,25 & $0,32(0,20-0,44)$ \\
\hline $30-39$ & 0,40 & 0,24 & 0,20 & 0,18 & 0,17 & 0,13 & $0,22(0,12-0,32)$ \\
\hline $40-59$ & 0,27 & 0,45 & 0,39 & 0,34 & 0,36 & 0,20 & $0,33(0,24-0,43)$ \\
\hline $60+$ & 0,55 & 0,60 & 0,55 & 0,51 & 0,32 & 0,51 & $0,51(0,40-0,61)$ \\
\hline
\end{tabular}


Au cours de la période de l'étude, les taux d'incidence moyens standardisés en fonction de l'âge étaient de 0,63 (IC : 0,50-0,75) chez les hommes et de 0,57 (IC:0,42-0,73) chez les femmes, ce qui constitue un écart non significatif.

\section{Caractère saisonnier}

Au total, 99 cas pour lesquels la date d'apparition était manquante ont été exclus aux fins de l'analyse saisonnière. L'incidence de cas de méningococcie invasive a été la plus élevée au cours des mois d'hiver. Le nombre moyen mensuel de cas était nettement plus élevé ( $p<0,0001)$ en hiver (18 cas) qu'en été (12 cas).

\section{Répartition par sous-types}

\section{Sérogroupe}

Au total, des données relatives aux sérogroupes étaient disponibles pour 1092 cas (93\%). Le nombre de cas signalés était le plus élevé pour le sérogroupe B (669), suivi des sérogroupes Y (196), C (138) et W-135 (62). Les cas relevant de ces quatre sérogroupes représentaient $91 \%$ du total de cas signalés de méningococcie invasive. Les cas de méningococcie invasive attribuables à d'autres sérogroupes étaient rares et comprenaient les sérogroupes $29 \mathrm{E}$ ( 7 cas), A ( 3 cas), X ( 2 cas) et $Z$ ( 2 cas); 10 cas étaient non sérogroupables et 3 étaient non précisés.

Le Tableau 1 et la Figure 4 fournissent la répartition détaillée de sérogroupes de cas de méningococcie invasive au Canada. Le taux d'incidence moyen standardisé en fonction de l'âge pour le sérogroupe B était le plus élevé $(0,36, I C: 0,29-0,43)$ de tous les sérogroupes. Le taux d'incidence ajuste pour le sérogroupe $B$ en $2010(0,24$, IC : $0,19-0,30$ ) était nettement inférieur à 2006, 2007 et 2009. La proportion de cas du sérogroupe $B$ parmi tous les cas de méningococcie invasive a été la plus élevée, au cours de toutes les années de la période d'étude, et a représenté de $50 \%$ à $62 \%$ des cas. Le taux d'incidence standardisé en fonction de l'âge de cas du sérogroupe C a nettement diminué de 0,14 (IC : 0,10-0,19) en 2006 à $0,01(0,004-0,04)$ en 2011. La proportion de cas du sérogroupe $C$ était nettement plus élevée que pour le sérogroupe $W-135$, de $2006(p<0,001)$ à $2008(p=0,02)$; la différence était négligeable de 2009 à 2011. La proportion de cas du sérogroupe $C$ est devenue nettement plus faible que pour le sérogroupe $Y$ en $2010(p=0,004)$ et en $2011(p<0,0001)$. Même si la proportion de cas du sérogroupe $Y$ a augmenté progressivement de $13 \%$ en 2006 à $21 \%$ en 2011, les taux d'incidence standardisés en fonction de l'âge pour ce groupe n'ont pas fluctué de façon importante au cours de la période de l'étude (de $0,07$ à 0,10$)$. La proportion de cas du sérogroupe $Y$ est demeurée nettement plus élevée que pour le sérogroupe $W-135$, de 2006 à 2011. Le taux d'incidence standardisé en fonction de l'âge de cas du sérogroupe W-135 a fluctué de 0,02 à 0,04, et aucune différence importante n'a été constatée au cours des six années.

Figure 4 : Proportion des sérogroupes parmi tous les cas signalés au Canada, par année, de 2006 à 2011*

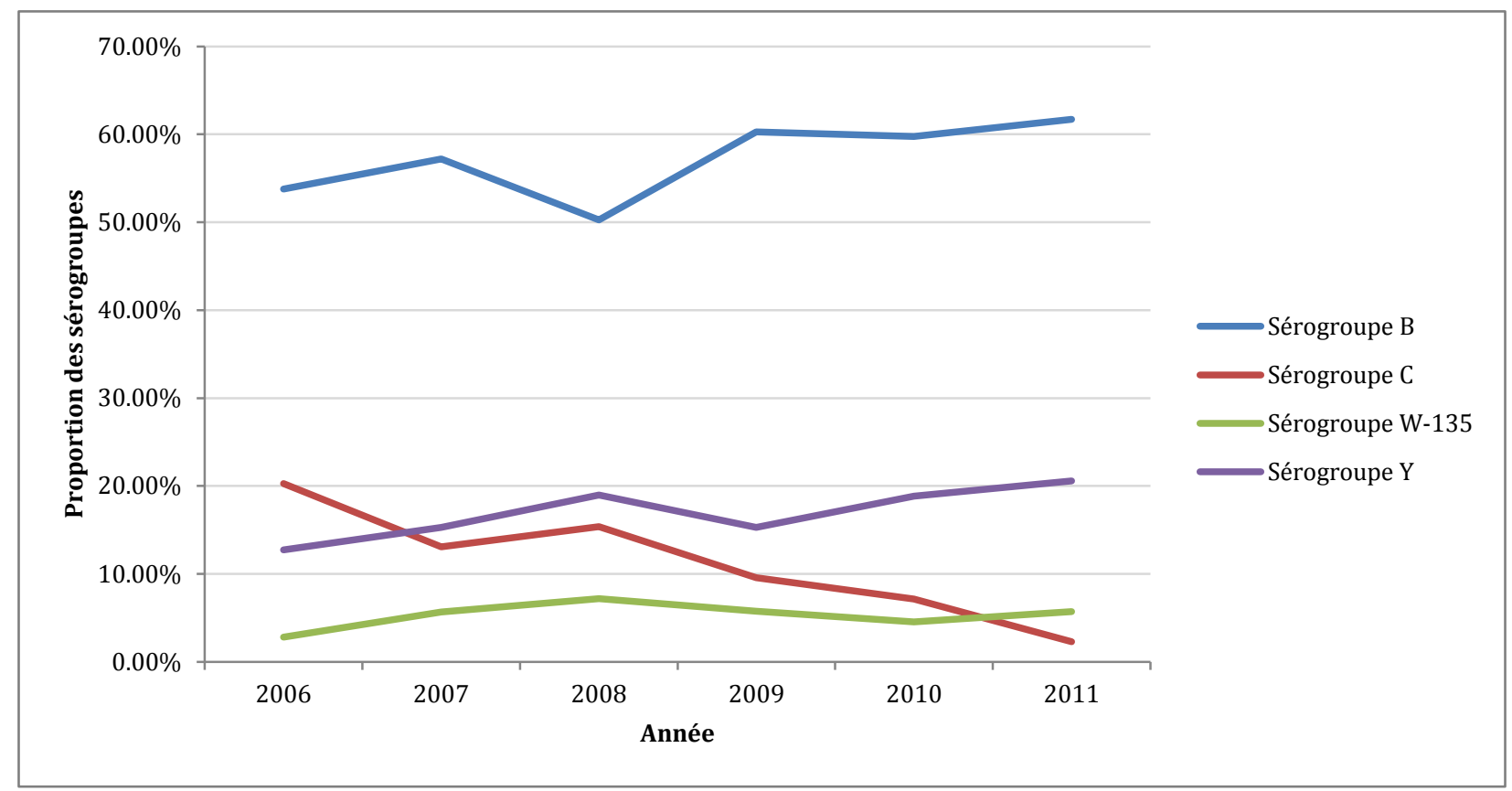

*Au total, 82 cas pour lesquels des données sur les sérogroupes étaient manquantes ont été exclus. 


\section{Sérotypes et sous-types sérologiques}

L'analyse des sérotypes et des sous-types sérologiques a inclus $73 \%$ des cas de méningococcie invasive (862/1 174), pour lesquels de l'information était disponible. Des 507 cas attribuables au sérogroupe B, $32 \%$ appartenaient au sérotype 17 , et $22 \%$ au sérotype $4 ; 14 \%$ n'ont pu être associés à un sérotype particulier. Les formules antigéniques les plus communes pour le sérogroupe B étaient B:17:P1.19 (25 \%) et B:4:P1.4 (9\%). Parmi les 113 cas du sérogroupe C, la majorité appartenaient au sérotype 2a (83\%), et $12 \%$ n'ont pu être associés à un sérotype particulier. La formule antigénique la plus commune pour le sérogroupe $C$ était C:2a:P1.2,5 (30\%). Sur les 163 cas du sérogroupe Y, $29 \%$ n'ont pu être associés à un sérotype, $28 \%$ appartenaient au sérotype $2 \mathrm{c}$ et $25 \%$ aux sérotypes 14 et 19 . Les formules antigéniques les plus communes pour le sérogroupe $Y$ étaient $Y: 14,19: P 1$.- (17\%) et $Y: 2 c: P 1.2,5$. (16\%). Parmi les 60 cas du sérogroupe W-135, 57 $(95 \%)$ n'ont pu être associés à un sérotype particulier, et les 3 autres cas appartenaient aux sérotypes 19, 2a et 2c.

\section{Caractéristiques cliniques}

\section{Diagnostics cliniques}

Au cours de la période de l'étude, sur les 375 cas avec diagnostics cliniques, $46 \%$ avaient la méningite et $45 \%$ la septicémie, avec ou sans manifestations autres. II y a eu six cas d'arthrite purulente.

\section{Décès}

II y a eu 94 décès au cours de la période de l'étude. Les taux de mortalité sont passés de 4,3\% en 2010 à $14,3 \%$ en 2011, pour une moyenne de $8,1 \%$. L'âge médian des cas de décès, par année, a varié de 17 à 26 ans, sauf en 2008, où il était de 45 ans. Aucune différence d'âge statistique n'a été constatée $(p=0,11)$. Les taux de mortalité pour les sérogroupes B, C, W-135 et Y étaient de 5,5\% (IC : 4,0-7,5\%), 14,5\% (IC : 9,6-21,3\%), 8,1\% (IC : 3,5-17,5\%) et 11,2\% (IC : 7,5-16,4\%), respectivement. Une différence importante de taux de mortalité a été constatée entre le sérogroupe B et le sérogroupe C.

\section{Mortalité}

Le taux de mortalité standardisé en fonction de l'âge associé à la méningococcie invasive a varié de 0,02 en 2010 à 0,07 en 2011, avec une moyenne de 0,05 . Les nourrissons ont présenté le taux de mortalité le plus élevé, soit de 0,62 (IC : 0,30-0,96).

\section{Analyse}

La présente étude montre que la méningococcie invasive est toujours endémique au Canada. Bien que les personnes de tout âge puissent être touchées, les nourrissons de moins de 1 an sont les plus à risque, suivis des enfants de 1 à 4 ans et des personnes de 15 à 19 ans. L'incidence de la méningococcie invasive est caractérisée par des variations saisonnières typiques, et un pic en hiver. Au Canada, les sérogroupes $B, C, W-135$ et $Y$ étaient les plus communément signalés. Ces constatations sont conformes aux précédentes données pour le Canada $(8,17,22)$. Grâce à l'introduction de programmes d'immunisation systématique des enfants et des adolescents (7), l'incidence du sérogroupe $C$ a nettement diminué, non sans surprise, au cours de la période de l'étude. Depuis 2007, tous les territoires et provinces du Canada offrent des programmes d'immunisation systématique des enfants contre l'infection au méningocoque $C$ (7) dans le cadre de leurs programmes d'immunisation de routine. Parallèlement à la période de mise en œuvre précoce (2002-2005) des programmes d'immunisation contre le méningocoque $C$, l'incidence moyenne du sérogroupe $C$ a diminué de $39 \%$, soit de 0,18 (8) à 0,07 . Le sérogroupe B est la principale cause de méningococcie invasive, et les taux d'incidence de maladies associées aux sérogroupes $\mathrm{W}-135$ et $\mathrm{Y}$ se sont stabilisés à des niveaux relativement plus bas. Le Comité consultatif national de l'immunisation a recommandé l'utilisation de vaccins quadrivalents conjugués contre le méningocoque pour les groupes à haut risque âgés de 2 à 55 ans, en 2007, ainsi que des programmes d'immunisation systématique des adolescents à l'aide d'un vaccin conjugué contre le méningocoque $C$, ou d'un vaccin quadrivalent conjugué contre le méningocoque en 2009 (7). Au cours de la période de l'étude, seuls quelques territoires et provinces avaient mis en œuvre des programmes d'immunisation à l'aide de vaccins quadrivalents conjugués contre le méningocoque. Par conséquent, la présente étude n'est pas en mesure d'évaluer l'incidence des programmes d'immunisation pour ce qui est du vaccin quadrivalent conjugué contre le méningocoque. Des données de surveillance accrue sont requises pour d'autres années. 
Des études biologiques de la population de souches de $N$. meningitidisinvasives par typage génomique multilocus ont révélé l'existence d'un clone prédominant du sérogroupe B ST-269 dans la province du Québec, menant à une augmentation de l'incidence de la méningococcie invasive (23-25). Dans la province voisine de l'Ontario, aucune augmentation similaire de cette souche ou de cas de maladie n'a été observée $(26,27)$. Une modification inhabituelle du génotype de souches de méningococcie invasive du groupe $\mathrm{C}$ a également été observée, de façon prédominante, du type ET15 au type ET-37 (non-ET-15) (28). Le taux de létalité global de la méningococcie invasive au Canada est demeuré stable par rapport aux années précédentes $(8,17)$, et s'avère comparable à celui d'autres nations $(29,30)$. La méningococcie invasive causée par le sérogroupe $C$ était beaucoup plus sévère que celle causée par le sérogroupe $B$, sur le plan de la létalité. La répartition des sérotypes et sous-types sérologiques des souches de $N$. meningitidis dans le cadre de la présente étude était similaire à celle des cas signalés dans les années précédentes (8), selon un nombre limité d'échantillons.

En conclusion, selon des données nationales de surveillance améliorée, après la mise en œuvre de programmes d'immunisation systématique des enfants à l'aide de vaccins conjugués monovalents contre le méningocoque du groupe $C$ dans l'ensemble des provinces et territoires (depuis 2007), l'incidence du méningocoque du groupe $C$ se trouve actuellement à son plus bas niveau historique. Compte tenu de l'ajout de vaccins quadrivalents conjugués contre le méningocoque, l'on pourrait s'attendre à une réduction supérieure de l'incidence d'infections à méningocoque au Canada. Des études subséquentes mettant l'accent sur l'incidence des différents programmes de vaccination contre le méningocoque au Canada sont requises.

\section{Limites}

Le Système national de surveillance améliorée de la méningococcie invasive ne reçoit pas les données des provinces et territoires en temps réel, et les cas signalés à l'échelle nationale ne sont pas liés aux données de laboratoire et épidémiologiques. En outre, certains éléments de données étaient incomplets, puisqu'il n'existe pas d'entente officielle de partage des données. Par conséquent, il n'est pas possible de déterminer si la présente étude a sous-estimé ou surestimé les caractéristiques de cas. Les cas faisant partie d'une éclosion n'ont pas été définis comme tels, dans cette étude, en raison du manque de données détaillées fournies par les provinces et territoires. Le statut d'immunisation des cas n'a pas été analysé, car les antécédents vaccinaux étaient manquants dans plus de la moitié des cas. En raison du manque de fiabilité des résultats fondés sur un petit nombre, il convient de faire preuve de prudence dans l'interprétation des résultats, comme les taux de mortalité et les taux d'incidence propre à des sérogroupes particuliers, basés sur moins de 20 cas.

\section{Remarques et remerciements}

Les données extraites du Système national de surveillance améliorée de la méningococcie invasive représentent un aperçu au moment de leur extraction, et peuvent différer des données avancées dans des publications précédentes ou subséquentes. Nous remercions les ministères de la Santé ainsi que les laboratoires des provinces et territoires pour les données fournies au Système national de surveillance améliorée de la méningococcie invasive. Nous désirons également remercier tous les collègues des ministères de la Santé des provinces et territoires pour les commentaires fournis dans le cadre du présent rapport.

\section{Conflit d'intérêts}

II n'y a aucun conflit d'intérêts à déclarer.

\section{Références}

(1) Heymann DL. Control of Communicable Diseases Manual. 19th ed. American Public Health Association; 2008. p. 403.

(2) Mandell G, Bennett J, Dolin P. Chapter 211 - Neisseria meningitidis. Mandell, Douglas, and Bennett's Principles and Practice of Infectious Diseases. 7th ed. Philadelphia: Churchill Livingstone; 2009. 
(3) Agence de la santé publique du Canada. Définitions nosologiques des maladies transmissibles faisant l'objet d'une surveillance nationale. Relevé des maladies transmissibles au Canada, vol. 35(S2), novembre 2009.

(4) Varughese PV, Acres S. Meningococcal disease in Canada and serogroup distribution. Can Dis Wkly Rep 1983;9(45):177-80.

(5) Whalen CM, Hockin JC, Ryan A, Ashton F. The changing epidemiology of invasive meningococcal disease in Canada, 1985 through 1992: emergence of a virulent clone of Neisseria meningitidis. JAMA 1995 February 1;273(5):390-394.

(6) Comité consultatif national de l'immunisation (CCNI). Une déclaration d'un comité consultatif (DCC). Déclaration sur l'utilisation recommandée des vaccins antiméningococciques. Relevé des maladies transmissibles au Canada, vol. 27, p. 2-36, octobre 2001.

(7) Comité consultatif national de l'immunisation (CCNI). Mise à jour des recommandations concernant la méningococcie et le vaccin conjugué contre le méningocoque. Une déclaration d'un comité consultatif (DCC). Relevé des maladies transmissibles au Canada, vol. 35 (DCC-3), p. 1-40, avril 2009.

(8) Surveillance accrue des méningococcies invasives au Canada, du $1^{\mathrm{er}}$ janvier 2004 au 31 décembre 2005. Relevé des maladies transmissibles au Canada, vol. 33, $n^{\circ} 10$, p. 1-15, $1^{\mathrm{er}}$ juin 2007.

(9) Ashton FE, Ryan JA. Association of serotypes 2A and 2B with meningococcal disease in Canada. Can Dis Wkly Rep 1989 Mar 18;15(11):63-65.

(10) Varughese PV, Carter AO. Meningococcal disease in Canada. Surveillance summary to 1987. Can Dis Wkly Rep 1989 Apr 29;15(17):89-96.

(11) Meningococcal disease - Canada, 1991. Can Dis Wkly Rep 1991 Dec 28;17(52):293.

(12) Ashton FE, Mancino L, Ryan AJ, Poolman JT, Abdillahi H, Zollinger WD. Serotypes and subtypes of Neisseria meningitidis serogroup B strains associated with meningococcal disease in Canada, 1977-1989. Can J Microbiol 1991 Aug;37(8):613-617.

(13) Bureau de l'épidémiologie des maladies transmissibles, Laboratoire de lutte contre la maladie. Lignes directrices pour la lutte contre les atteintes méningococciques. Relevé des maladies transmissibles au Canada, vol. 20, n 3, p. 17-27, 15 février 1994.

(14) Squires, S.G., Pelletier, L., Mungai, M., Tsang, R., Collins, F., Stoltz, J. Les méningococcies invasives au Canada, du $1^{\text {er }}$ janvier 1997 au 31 décembre 1998. Relevé des maladies transmissibles au Canada, vol. 26, $\mathrm{n}^{\circ} 21$, p. 177-182, $1^{\text {er }}$ novembre 2000.

(15) Squires, S.G., Deeks, S.L., Tsang, R.S. Surveillance accrue des méningococcies invasives au Canada : du $1^{\mathrm{er}}$ janvier 1999 au 31 décembre 2001. Relevé des maladies transmissibles au Canada, vol. 30, $\mathrm{n}^{\circ} 3, \mathrm{p} .17-$ 28, $1^{\text {er }}$ février 2004.

(16) Law DK, Stoltz J, Henderson AM, Tsang RS. Antigenic and genetic characterization of serogroup C meningococci isolated from invasive meningococcal disease cases in Canada from 1999 to 2003. Can J Microbiol $2005 \mathrm{Jul} ; 51(7): 523-530$.

(17) Surveillance accrue des méningococcies invasives au Canada : du $1^{\mathrm{er}}$ janvier 2002 au 31 décembre 2003. Relevé des maladies transmissibles au Canada, vol. 32, nº 8, p. 97-107, 15 avril 2006.

(18) Tsang RS, Law DK, Henderson AM, Blake ML, Stoltz J. Increase in serogroup C meningococcal disease in Canada is associated with antigenic changes in the protein antigens of the ET-15 clone of Neisseria meningitidis. J Infect Dis 2006 Dec 15;194(12):1791-2; author reply 1792-3.

(19) Statistique Canada, Division de la démographie, Section des estimations démographiques. Estimations de la population pour juillet, 2011. Estimations intercensitaires finales. 
(20) Statistique Canada. Tableau 105-0503 - Profil d'indicateurs de la santé, taux normalisés selon l'âge, estimations annuelles, selon le sexe, Canada, provinces et territoires, occasionnel, CANSIM (base de données). 2013. Accès : http://www5.statcan.gc.ca/cansim/pick-

choisir?id=1050503\&p2=33\&retrLang=fra\&lang=fra [consulté le 13 janvier 2014].

(21) Fay MP, Feuer EJ. Confidence intervals for directly standardized rates: a method based on the gamma distribution. Stat Med 1997 Apr 15;16(7):791-801.

(22) Bettinger JA, Scheifele DW, Le Saux N, Halperin SA, Vaudry W, Tsang R, et al. The impact of childhood meningococcal serogroup C conjugate vaccine programs in Canada. Pediatr Infect Dis J 2009 Mar;28(3):220224.

(23) Law DK, Lorange M, Ringuette L, Dion R, Giguere M, Henderson AM, et al. Invasive meningococcal disease in Quebec, Canada, due to an emerging clone of ST-269 serogroup B meningococci with serotype antigen 17 and serosubtype antigen P1.19 (B:17:P1.19). J Clin Microbiol 2006 Aug;44(8):2743-2749.

(24) Zhou J, Lefebvre B, Deng S, Gilca R, Deceuninck G, Law DK, et al. Invasive serogroup B Neisseria meningitidis in Quebec, Canada, 2003 to 2010: persistence of the ST-269 clone since it first emerged in 2003. J Clin Microbiol 2012 May;50(5):1545-1551.

(25) Gilca R, Deceuninck G, Lefebvre B, Tsang R, Amini R, Gilca V, et al. The changing epidemiology of meningococcal disease in Quebec, Canada, 1991-2011: potential implications of emergence of new strains. PLoS One 2012;7(11):e50659.

(26) Dang V, Jamieson FB, Wilson S, Rawte P, Crowcroft NS, Johnson K, et al. Epidemiology of serogroup B invasive meningococcal disease in Ontario, Canada, 2000 to 2010. BMC Infect Dis 2012 Aug 29;12:202.

(27) Jamieson FB, Rawte P, Deeks SL, Zhou J, Law DK, Deng S, et al. Genetic and antigenic characterization of invasive endemic serogroup B Neisseria meningitidis from Ontario, Canada, in 2001-2010. J Med Microbiol 2013 Jan;62(Pt 1):46-55.

(28) Zhou J, Jamieson F, Dolman S, Hoang LM, Rawte P, Tsang RS. Genetic and antigenic analysis of invasive serogroup C Neisseria meningitidis in Canada: a decrease in the electrophoretic type (ET)-15 clonal type and an increase in the proportion of isolates belonging to the ET-37 (but not ET-15) clonal type during the period from 2002 to 2009. Can J Infect Dis Med Microbiol 2012 Fall;23(3):e55-9.

(29) Thigpen MC, Whitney CG, Messonnier NE, Zell ER, Lynfield R, Hadler JL, et al. Bacterial meningitis in the United States, 1998-2007. N Engl J Med 2011 May 26;364(21):2016-2025.

(30) Hellenbrand W, Elias J, Wichmann O, Dehnert M, Frosch M, Vogel U. Epidemiology of invasive meningococcal disease in Germany, 2002-2010, and impact of vaccination with meningococcal C conjugate vaccine. J Infect 2013 Jan;66(1):48-56. 